\title{
Browder and Göhde fixed point theorem for monotone nonexpansive mappings
}

\author{
Buthinah Abdullatif Bin Dehaish ${ }^{1 *}$ and Mohamed Amine Khamsi' ${ }^{2,3}$
}

\author{
"Correspondence: \\ bbindehaish@yahoo.com \\ 'Department of Mathematics, \\ Faculty of Science-Al Faisaliah \\ Campus, King Abdulaziz University, \\ Jeddah, 21593, Saudi Arabia \\ Full list of author information is \\ available at the end of the article
}

\begin{abstract}
Let $X$ be a Banach space or a complete hyperbolic metric space. Let $C$ be a nonempty, bounded, closed, and convex subset of $X$ and $T: C \rightarrow C$ be a monotone nonexpansive mapping. In this paper, we show that if $X$ is a Banach space which is uniformly convex in every direction or a uniformly convex hyperbolic metric space, then $T$ has a fixed point. This is the analog to Browder and Göhde's fixed point theorem for monotone nonexpansive mappings.
\end{abstract}

MSC: Primary 46B20; 45D05; secondary 47E10; 34A12

Keywords: fixed point; hyperbolic metric spaces; Krasnoselskii iteration; monotone mapping; nonexpansive mapping; partially ordered; uniformly convex

\section{Introduction}

'The theory of fixed points is one of the most powerful tools of modern mathematics' said Felix Browder, who gave a new impetus to the modern fixed point theory via the development of nonlinear functional analysis as an active and vital branch of mathematics. The flourishing field of fixed point theory started in the early days of topology (the work of Poincaré, Lefschetz-Hopf, and Leray-Schauder). For example, the existence problems are usually translated into a fixed point problem like the existence of solutions to elliptic partial differential equations, or the existence of closed periodic orbits in dynamical systems, and more recently the existence of answer sets in logic programming.

Recently a new direction has been discovered dealing with the extension of the Banach contraction principle [1] to metric spaces endowed with a partial order. Ran and Reurings [2] successfully carried out the first attempt see also [3]. In particular, they showed how this extension is useful when dealing with some special matrix equations. A similar approach was carried out by Nieto and Rodríguez-López [4] and used such arguments in solving some differential equations. In [5] Jachymski gave a more general unified version of these extensions by considering graphs instead of a partial order. In this paper, we investigate the existence of fixed points of monotone nonexpansive mappings. In particular, we prove that if $X$ is a uniformly convex hyperbolic metric space, then any monotone nonexpansive mapping defined on a nonempty bounded convex subset has a fixed points.

In terms of content, this paper overlaps in places with the popular books on fixed point theory by Aksoy and Khamsi [6], by Goebel and Kirk [7], by Dugundji and Granas [8], by Khamsi and Kirk [9], and by Zeidler [10]. Material on the general theory of Banach

(c) 2016 Bin Dehaish and Khamsi. This article is distributed under the terms of the Creative Commons Attribution 4.0 International License (http://creativecommons.org/licenses/by/4.0/), which permits unrestricted use, distribution, and reproduction in any medium, provided you give appropriate credit to the original author(s) and the source, provide a link to the Creative Commons license, and indicate if changes were made. 
space geometry and hyperbolic geometry is drawn from many sources but the books by Beauzamy [11], by Diestel [12], by Goebel and Reich [13], and by Bridson and Haefliger [14] are worth of special mention.

\section{Preliminaries}

The extension of the Banach contraction principle in metric spaces endowed with a partial order was initiated by Ran and Reurings [2] (see also [15-18]). In order to discuss such extension, we will need to assume that the metric space $(M, d)$ is endowed with a partial order $\preceq$. We will say that $x, y \in M$ are comparable whenever $x \preceq y$ or $y \preceq x$. Next we give the definition of monotone mappings.

Definition 2.1 Let $(M, d, \preceq)$ be a metric space endowed with a partial order. Let $T: M \rightarrow$ $M$ be a map. $T$ is said to be monotone or order-preserving if

$$
x \preceq y \quad \Rightarrow \quad T(x) \preceq T(y),
$$

for any $x, y \in M$.

Next we give the definition of monotone Lipschitzian mappings.

Definition 2.2 Let $(M, d, \preceq)$ be a metric space endowed with a partial order. Let $T: M \rightarrow$ $M$ be a map. $T$ is said to be monotone Lipschitzian mapping if $T$ is monotone and there exists $k \geq 0$ such that

$$
d(T(x), T(y)) \leq k d(x, y)
$$

for any $x, y \in M$ such that $x$ and $y$ are comparable. If $k<1$, then we say that $T$ is a monotone contraction mapping. And if $k=1, T$ is called a monotone nonexpansive mapping. A point $x \in M$ is said to be a fixed point of $T$ whenever $T(x)=x$. The set of fixed points of $T$ will be denoted by Fix $(T)$.

Note that monotone Lipschitzian mappings are not necessarily continuous. They usually have a good topological behavior of comparable elements but not the entire set on which they are defined.

\section{Monotone nonexpansive mappings in hyperbolic metric spaces}

The fixed point theory for nonexpansive mappings finds its root in the works of Browder [19], Göhde [20], and Kirk [21] published in the same year 1965. Basically it took four decades to extend the contractive condition to the case of mappings with Lipschitz constant $k=1$. It was clear from the start that such mappings have a different behavior from contraction mappings. The first results obtained in 1965 were discovered in Banach spaces. It took a few decades to extend the fixed point theory of nonexpansive mappings to nonlinear domains. Similarly and following the extension of the Banach contraction principle to the case of metric spaces endowed with a partial order, it was natural to try to investigate the case of nonexpansive mappings into such metric spaces.

In this section, we will establish Browder and Göhde' s fixed point theorem for monotone nonexpansive mappings. The setting will be uniformly convex hyperbolic metric spaces. 
Let $(X, d)$ be a metric space. Suppose that there exist a family $\mathcal{F}$ of metric segments such that any two points $x, y$ in $X$ are endpoints of a unique metric segment $[x, y] \in \mathcal{F}([x, y]$ is an isometric image of the real line interval $[0, d(x, y)])$. We shall denote by $\beta x \oplus(1-\beta) y$ the unique point $\mathrm{z}$ of $[x, y]$ which satisfies

$$
d(x, z)=(1-\beta) d(x, y) \quad \text { and } \quad d(z, y)=\beta d(x, y)
$$

where $\beta \in[0,1]$. Such metric spaces with a family $\mathcal{F}$ of metric segments are usually called convex metric spaces [22]. Moreover, if we have

$$
d(\alpha p \oplus(1-\alpha) x, \alpha q \oplus(1-\alpha) y) \leq \alpha d(p, q)+(1-\alpha) d(x, y),
$$

for all $p, q, x, y$ in $X$, and $\alpha \in[0,1]$, then $X$ is said to be a hyperbolic metric space (see [23]). Obviously, normed linear spaces are hyperbolic spaces. As nonlinear examples, one can consider the Hadamard manifolds [24], the Hilbert open unit ball equipped with the hyperbolic metric [13], and the CAT(0) spaces [25-27]. We will say that a subset $C$ of a hyperbolic metric space $X$ is convex if $[x, y] \subset C$ whenever $x, y$ are in $C$.

Definition 3.1 Let $(M, d)$ be a hyperbolic metric space. We say that $M$ is uniformly convex (in short, UC) if for any $a \in M$, for every $r>0$, and for each $\epsilon>0$

$$
\delta(r, \varepsilon)=\inf \left\{1-\frac{1}{r} d\left(\frac{1}{2} x \oplus \frac{1}{2} y, a\right) ; d(x, a) \leq r, d(y, a) \leq r, d(x, y) \geq r \varepsilon\right\}>0 .
$$

From now onwards we assume that $M$ is a hyperbolic metric space and if $(M, d)$ is uniformly convex, then for every $s \geq 0, \varepsilon>0$, there exists $\eta(s, \varepsilon)>0$ depending on $s$ and $\epsilon$ such that

$$
\delta(r, \varepsilon)>\eta(s, \varepsilon)>0 \quad \text { for any } r>s .
$$

\section{Remark 3.1 [28]}

(i) Let us observe that $\delta(r, 0)=0$, and $\delta(r, \varepsilon)$ is an increasing function of $\varepsilon$ for every fixed $r$.

(ii) For $r_{1} \leq r_{2}$ we have

$$
1-\frac{r_{2}}{r_{1}}\left(1-\delta\left(r_{2}, \varepsilon \frac{r_{1}}{r_{2}}\right)\right) \leq \delta\left(r_{1}, \varepsilon\right) .
$$

(iii) If $(M, d)$ is uniformly convex, then $(M, d)$ is strictly convex, i.e., whenever

$$
d\left(\frac{1}{2} x \oplus \frac{1}{2} y, a\right)=d(x, a)=d(y, a)
$$

for any $x, y, a \in M$, then we must have $x=y$.

Among the nice properties satisfied by uniformly convex hyperbolic metric space $(X, d)$ is the property (R) [29] which says that if $\left\{C_{n}\right\}$ is a decreasing sequence of nonempty, bounded, convex, and closed subsets of $X$, then $\bigcap_{n \geq 1} C_{n} \neq \emptyset$. The following technical lemma will be useful. 
Lemma 3.1 Let $C$ be a nonempty closed convex subset of uniformly convex hyperbolic metric space $(X, d)$. Let $\tau: C \rightarrow[0,+\infty)$ be a type function, i.e., there exists a bounded sequence $\left\{x_{n}\right\} \in X$ such that

$$
\tau(x)=\limsup _{n \rightarrow+\infty} d\left(x_{n}, x\right)
$$

for any $x \in C$. Then $\tau$ is continuous. Since $X$ is hyperbolic, $\tau$ is convex, i.e., the subset $\{x \in C ; \tau(x) \leq r\}$ is convex for any $r \geq 0$. Moreover, there exists a unique minimum point $z \in C$ such that

$$
\tau(z)=\inf \{\tau(x) ; x \in C\} .
$$

Proof The continuity and convexity of $\tau$ are obvious. Let us show the existence of the minimum point of $\tau$. Set $\tau_{0}=\inf \{\tau(x) ; x \in C\}$. Then for any $n \geq 1$, the subset $C_{n}=$ $\left\{x \in C ; \tau(x) \leq \tau_{0}+1 / n\right\}$ is not empty and is a closed convex subset of $C$. The property (R) will then imply that $C_{\infty}=\bigcap_{n \geq 1} C_{n} \neq \emptyset$. Clearly we have $C_{\infty}=\left\{z \in C ; \tau(z)=\tau_{0}\right\}$. Let us prove that $C_{\infty}$ is reduced to one point. Let $z_{1}$ and $z_{2}$ be in $C_{\infty}$. Assume that $z_{1} \neq z_{2}$. In this case, we must have $\tau_{0} \neq 0$. Let $\alpha \in\left(0, \tau_{0}\right)$. Then there exists $n_{0} \geq 1$ such that for any $n \geq n_{0}$ we have

$$
d\left(x_{n}, z_{1}\right) \leq \tau_{0}+\alpha \quad \text { and } \quad d\left(x_{n}, z_{2}\right) \leq \tau_{0}+\alpha .
$$

Since $d\left(z_{1}, z_{2}\right) \geq\left(\tau_{0}+\alpha\right) d\left(z_{1}, z_{2}\right) / 2 \tau_{0}$,

$$
d\left(x_{n}, \frac{1}{2} z_{1} \oplus \frac{1}{2} z_{2}\right) \leq\left(\tau_{0}+\alpha\right)\left(1-\delta\left(\tau_{0}+\alpha, \frac{d\left(z_{1}, z_{2}\right)}{2 \tau_{0}}\right)\right),
$$

which implies

$$
d\left(x_{n}, \frac{1}{2} z_{1} \oplus \frac{1}{2} z_{2}\right) \leq\left(\tau_{0}+\alpha\right)\left(1-\eta\left(\tau_{0}, \frac{d\left(z_{1}, z_{2}\right)}{2 \tau_{0}}\right)\right) .
$$

If we let $n \rightarrow+\infty$, we get

$$
\tau\left(\frac{1}{2} z_{1} \oplus \frac{1}{2} z_{2}\right) \leq\left(\tau_{0}+\alpha\right)\left(1-\eta\left(\tau_{0}, \frac{d\left(z_{1}, z_{2}\right)}{2 \tau_{0}}\right)\right) .
$$

Finally let $\alpha \rightarrow 0$ to get

$$
\tau\left(\frac{1}{2} z_{1} \oplus \frac{1}{2} z_{2}\right) \leq \tau_{0}\left(1-\eta\left(\tau_{0}, \frac{d\left(z_{1}, z_{2}\right)}{2 \tau_{0}}\right)\right) .
$$

This is a contradiction with the fact that $C_{\infty}$ is convex which implies

$$
\tau\left(\frac{1}{2} z_{1} \oplus \frac{1}{2} z_{2}\right)=\tau_{0}<\tau_{0}
$$

We have noted before that monotone Lipschitzian mappings may not be continuous because they fail to have nice global behavior. For this reason, one approach to study such 
mappings is to use iterative methods. Indeed, let $(X, d)$ be a hyperbolic metric space endowed with a partial order $\preceq$. Throughout, we will assume that order intervals are convex and closed. Recall that an order interval is any of the subsets $[a, \rightarrow)=\{x \in X ; a \preceq x\}$ and $(\leftarrow, b]=\{x \in X ; x \preceq b\}$, for any $a, b \in X$. Let $C$ be a nonempty convex subset of $X$ not reduced to one point. Let $T: C \rightarrow C$ be monotone nonexpansive mapping. Fix $\lambda \in(0,1)$ and $x_{0} \in C$. Consider the Krasnoselskii-Ishikawa $[30,31]$ iteration sequence $\left\{x_{n}\right\}$ in $C$ defined by

$$
x_{n+1}=(1-\lambda) x_{n} \oplus \lambda T\left(x_{n}\right), \quad n \geq 0 .
$$

Assume that $x_{0}$ and $T\left(x_{0}\right)$ are comparable. Without loss of any generality, we assume that $x_{0} \preceq T\left(x_{0}\right)$. Since order intervals are convex, we have $x_{1} \preceq x_{2} \preceq T\left(x_{1}\right)$. Since $T$ is monotone, we get $T\left(x_{1}\right) \preceq T\left(x_{2}\right)$. By induction, we will prove that

$$
x_{n} \preceq x_{n+1} \preceq T\left(x_{n}\right) \preceq T\left(x_{n+1}\right),
$$

for any $n \geq 1$, which implies, since $T$ is monotone nonexpansive,

$$
d\left(T\left(x_{n+1}\right), T\left(x_{n}\right)\right) \leq d\left(x_{n+1}, x_{n}\right) .
$$

In order to proceed, we will need the following fundamental result. Its origin may be found in $[7,32]$.

Proposition 3.1 Let $(X, d, \preceq)$ be a partially ordered hyperbolic metric space having the above properties. Let $C$ be a convex and bounded subset of $X$ not reduced to one point. Let $T: C \rightarrow C$ be a monotone nonexpansive mapping. Fix $\lambda \in(0,1)$ and $x_{0} \in C$ such that $x_{0}$ and $T\left(x_{0}\right)$ are comparable. Consider the sequence $\left\{x_{n}\right\}$ in $C$ defined by (KIS). Hence

$$
\begin{aligned}
(1+n \lambda) d\left(T\left(x_{i}\right), x_{i}\right) \leq & d\left(T\left(x_{i+n}\right), x_{i}\right) \\
& +(1-\lambda)^{-n}\left(d\left(T\left(x_{i}\right), x_{i}\right)-d\left(T\left(x_{i+n}\right), x_{i+n}\right)\right)
\end{aligned}
$$

for any $i, n \in \mathbb{N}$. Then we have

$$
\lim _{n \rightarrow+\infty} d\left(x_{n}, T\left(x_{n}\right)\right)=0
$$

i.e., $\left\{x_{n}\right\}$ is an approximate fixed point sequence of $T$.

Proof The first part of the proposition may be found in $[7,32]$ and is obtained by an induction argument. As for the second part, note that the sequence $\left\{d\left(x_{n+1}, x_{n}\right)\right\}$ is decreasing. This follows from the inequalities, which hold since $X$ is hyperbolic,

$$
\begin{aligned}
d\left(x_{n+2}, x_{n+1}\right) & =d\left((1-\lambda) x_{n+1} \oplus \lambda T\left(x_{n+1}\right),(1-\lambda) x_{n} \oplus \lambda T\left(x_{n}\right)\right) \\
& \leq(1-\lambda) d\left(x_{n+1}, x_{n}\right)+\lambda d\left(T\left(x_{n+1}\right), T\left(x_{n}\right)\right) \\
& \leq(1-\lambda) d\left(x_{n+1}, x_{n}\right)+\lambda d\left(x_{n+1}, x_{n}\right) \\
& =d\left(x_{n+1}, x_{n}\right)
\end{aligned}
$$


for any $n \geq 0$. Since $d\left(T\left(x_{n}\right), x_{n}\right)=d\left(x_{n+1}, x_{n}\right) /(1-\lambda)$, we conclude that the sequence $\left\{d\left(T\left(x_{n}\right), x_{n}\right)\right\}$ is also decreasing. Set $r=\lim _{n \rightarrow+\infty} d\left(T\left(x_{n}\right), x_{n}\right)$. Then if we let $i \rightarrow+\infty$ in the inequality (GK), we get $(1+n \lambda) r \leq \delta(C)$, for any $n \geq 1$, where $\delta(C)=\sup \{d(x, y)$; $x, y \in C\}<+\infty$. This will obviously imply $r=0$, i.e., $\lim _{n \rightarrow+\infty} d\left(x_{n}, T\left(x_{n}\right)\right)=0$.

Now we are ready to state the main result of this section.

Theorem 3.1 Let $(X, d, \preceq)$ be a partially ordered hyperbolic metric space as described above. Assume $(X, d)$ is uniformly convex. Let $C$ be a nonempty convex closed bounded subset of $X$ not reduced to one point. Let $T: C \rightarrow C$ be a monotone nonexpansive mapping. Assume there exists $x_{0} \in C$ such that $x_{0}$ and $T\left(x_{0}\right)$ are comparable. Then $T$ has a fixed point.

Proof Without loss of any generality, assume that $x_{0} \preceq T\left(x_{0}\right)$. Consider the KrasnoselskiiIshikawa sequence $\left\{x_{n}\right\}$ generated by (KIS) starting at $x_{0}$ with $\lambda \in(0,1)$. Since $X$ is uniformly convex, it satisfies the property (R). Using the properties of $\left\{x_{n}\right\}$, we know that

$$
C_{\infty}=\bigcap_{n \geq 0}\left[x_{n}, \rightarrow\right) \cap C=\bigcap_{n \geq 0}\left\{x \in C ; x_{n} \preceq x\right\} \neq \emptyset .
$$

Let $x \in C_{\infty}$, then $x_{n} \preceq x$ and since $T$ is monotone, we get $x_{n} \preceq T\left(x_{n}\right) \preceq T(x)$, for any $n \geq 0$, i.e., $T\left(C_{\infty}\right) \subset C_{\infty}$. Consider the type function $\tau: C_{\infty} \rightarrow[0,+\infty)$ generated by $\left\{x_{n}\right\}$, i.e., $\tau(x)=\limsup _{n \rightarrow+\infty} d\left(x_{n}, x\right)$. Since $\left\{x_{n}\right\}$ is an approximate fixed point sequence of $T$, we get $\tau(x)=\limsup _{n \rightarrow+\infty} d\left(T\left(x_{n}\right), x\right)$, for any $x \in C_{\infty}$. Lemma 3.1 implies the existence of a unique $z \in C_{\infty}$ such that $\tau(z)=\inf \left\{\tau(x) ; x \in C_{\infty}\right\}$. Since $z \in C_{\infty}$, we have $x_{n} \leq z$, for any $n \geq 1$, which implies

$$
\tau(T(z))=\limsup _{n \rightarrow+\infty} d\left(T\left(x_{n}\right), T(z)\right) \leq \limsup _{n \rightarrow+\infty} d\left(x_{n}, z\right)=\tau(z) .
$$

The uniqueness of the minimum point implies that $z=T(z)$, i.e., $z$ is a fixed point of $T$.

In the next section, we will show how to weaken the uniform convexity property when we assume that $X$ is a vector space.

\section{Monotone nonexpansive mappings in Banach spaces}

Let $(X,\|\cdot\|)$ be a Banach space. We say that $X$ is uniformly convex in the direction $z \in X$, with $\|z\|=1$, if $\delta(\varepsilon, z)>0$, where

$$
\delta(\varepsilon, z)=\inf \left\{1-\left\|\frac{x+y}{2}\right\| ;\|x\| \leq 1,\|y\| \leq 1, x-y=\alpha z \text {, and }\|x-y\| \geq \varepsilon\right\}
$$

for any $\varepsilon \in(0,2]$. Uniform convexity in every direction was introduced by Garkavi [33] in connection with his study of Chebyshev centers. Zizler [34] proved that any separable Banach space has an equivalent norm which is uniformly convex in every direction. It is also known that uniformly convex Banach spaces are super-reflexive [11] which shows that the class of uniformly convex is a lot smaller that the class of uniformly convex in every direction. We have a similar conclusion to Lemma 3.1 in Banach spaces which are uniformly convex in every direction. 
Lemma 4.1 Let $(X,\|\cdot\|)$ be a Banach space which is uniformly convex in every direction. Let $C$ be a weakly compact nonempty convex subset of $X$. Let $\tau: C \rightarrow[0,+\infty)$ be a type function. Then there exists a unique minimum point $z \in C$ such that

$$
\tau(z)=\inf \{\tau(x) ; x \in C\} .
$$

Proof Let $\left\{x_{n}\right\}$ be a bounded sequence such that $\tau(x)=\limsup _{n \rightarrow+\infty}\left\|x_{n}-x\right\|$, for $x \in C$. Note that the continuity and convexity of $\tau$ are obvious. Let us show the existence of the minimum point of $\tau$. Set $\tau_{0}=\inf \{\tau(x) ; x \in C\}$. Then for any $n \geq 1$, the subset $C_{n}=$ $\left\{x \in C ; \tau(x) \leq \tau_{0}+1 / n\right\}$ is not empty and is a closed convex subset of $C$. Since $C$ is weakly compact, $C_{\infty}=\bigcap_{n \geq 1} C_{n} \neq \emptyset$. Clearly we have $C_{\infty}=\left\{z \in C ; \tau(z)=\tau_{0}\right\}$. Let us prove that $C_{\infty}$ is reduced to one point. Let $z_{1}$ and $z_{2}$ be in $C_{\infty}$. Assume that $z_{1} \neq z_{2}$. In this case, we must have $\tau_{0} \neq 0$. Set $z=\left(z_{1}-z_{2}\right) /\left\|z_{1}-z_{2}\right\|$. Let $\alpha \in\left(0, \tau_{0}\right)$. Then there exists $n_{0} \geq 1$ such that for any $n \geq n_{0}$ we have

$$
\left\|x_{n}-z_{1}\right\| \leq \tau_{0}+\alpha \quad \text { and } \quad\left\|x_{n}-z_{2}\right\| \leq \tau_{0}+\alpha .
$$

Since $\left\|z_{1}-z_{2}\right\| \geq\left(\tau_{0}+\alpha\right)\left\|z_{1}-z_{2}\right\| / 2 \tau_{0}$,

$$
\left\|x_{n}-\frac{z_{1}+z_{2}}{2}\right\| \leq\left(\tau_{0}+\alpha\right)\left(1-\delta\left(\frac{\left\|z_{1}-z_{2}\right\|}{2 \tau_{0}}, z\right)\right)
$$

for any $n \geq n_{0}$. If we let $n \rightarrow+\infty$, we get

$$
\tau\left(\frac{z_{1}+z_{2}}{2}\right) \leq\left(\tau_{0}+\alpha\right)\left(1-\delta\left(\frac{\left\|z_{1}-z_{2}\right\|}{2 \tau_{0}}, z\right)\right) .
$$

Finally let $\alpha \rightarrow 0$ to get

$$
\tau\left(\frac{z_{1}+z_{2}}{2}\right) \leq \tau_{0}\left(1-\delta\left(\frac{\left\|z_{1}-z_{2}\right\|}{2 \tau_{0}}, z\right)\right) .
$$

This is a contradiction with the fact that $C_{\infty}$ is convex, which implies

$$
\tau\left(\frac{z_{1}+z_{2}}{2}\right)=\tau_{0}<\tau_{0}
$$

Since Banach spaces are hyperbolic metric spaces, we have a similar conclusion to Proposition 3.1.

Proposition 4.1 Let $(X,\|\cdot\|, \preceq)$ be a partially ordered Banach such that order intervals are closed and convex. Let $C$ be a convex and bounded subset of $X$ not reduced to one point. Let $T: C \rightarrow C$ be a monotone nonexpansive mapping. Fix $\lambda \in(0,1)$ and $x_{0} \in C$ such that $x_{0}$ and $T\left(x_{0}\right)$ are comparable. Consider the sequence $\left\{x_{n}\right\}$ in $C$ defined by (KIS). Hence

$$
\begin{aligned}
(1+n \lambda)\left\|T\left(x_{i}\right)-x_{i}\right\| \leq & \left\|T\left(x_{i+n}\right)-x_{i}\right\| \\
& +(1-\lambda)^{-n}\left(\left\|T\left(x_{i}\right)-x_{i}\right\|-\left\|T\left(x_{i+n}\right)-x_{i+n}\right\|\right),
\end{aligned}
$$

for any $i, n \in \mathbb{N}$. Then we have $\lim _{n \rightarrow+\infty}\left\|x_{n}-T\left(x_{n}\right)\right\|=0$, i.e., $\left\{x_{n}\right\}$ is an approximate fixed point sequence of $T$. 
Using the same ideas in the proof of Theorem 3.1, we get the following fixed point result.

Theorem 4.1 Let $(X,\|\cdot\|, \preceq)$ be a partially ordered Banach such that order intervals are closed and convex. Assume $X$ is uniformly convex in every direction. Let $C$ be a nonempty weakly compact convex subset of $X$ not reduced to one point. Let $T: C \rightarrow C$ be a monotone nonexpansive mapping. Assume there exists $x_{0} \in C$ such that $x_{0}$ and $T\left(x_{0}\right)$ are comparable. Then $T$ has a fixed point.

\section{Competing interests}

The authors declare that they have no competing interests.

\section{Authors' contributions}

All authors contributed equally to the writing of this paper. All authors read and approved the final manuscript.

\section{Author details}

${ }^{1}$ Department of Mathematics, Faculty of Science-Al Faisaliah Campus, King Abdulaziz University, Jeddah, 21593, Saudi Arabia. ${ }^{2}$ Department of Mathematical Sciences, University of Texas at El Paso, El Paso, TX 79968, USA. ${ }^{3}$ Department of Mathematics \& Statistics, King Fahd University of Petroleum and Minerals, Dhahran, 31261, Saudi Arabia.

\section{Acknowledgements}

This work was funded by the Deanship of Scientific Research (DSR), King Abdulaziz University, Jeddah. The authors, therefore, acknowledge with thanks technical and financial support of DSR.

Received: 26 August 2015 Accepted: 27 January 2016 Published online: 20 February 2016

\section{References}

1. Banach, S: Sur les opérations dans les ensembles abstraits et leurs applications. Fundam. Math. 3, 133-181 (1922)

2. Ran, ACM, Reurings, MCB: A fixed point theorem in partially ordered sets and some applications to matrix equations. Proc. Am. Math. Soc. 132(5), 1435-1443 (2004)

3. El-Sayed, SM, Ran, ACM: On an iteration method for solving a class of nonlinear matrix equations. SIAM J. Matrix Anal. Appl. 23(3), 632-645 (2002)

4. Nieto, JJ, Rodriguez-Lopez, R: Contractive mapping theorems in partially ordered sets and applications to ordinary differential equations. Order 22(3), 223-239 (2005)

5. Jachymski, J: The contraction principle for mappings on a metric space with a graph. Proc. Am. Math. Soc. 136 1359-1373 (2008)

6. Aksoy, AG, Khamsi, MA: Nonstandard Methods in Fixed Point Theory. Springer, New York (1990)

7. Goebel, K, Kirk, WA: Topics in Metric Fixed Point Theory. Cambridge University Press, Cambridge (1990)

8. Dugundji, J, Granas, A: Fixed Point Theory. Polska Akademia Nauk, Instytut Matematyczny, PWN-Polish Scientific Publ., Warszawa (1982)

9. Khamsi, MA, Kirk, WA: An Introduction to Metric Spaces and Fixed Point Theory. Wiley, New York (2001)

10. Zeidler, E: Nonlinear Functional Analysis and Its Applications I: Fixed-Point Theorems. Springer, New York (1986)

11. Beauzamy, B: Introduction to Banach Spaces and Their Geometry. North-Holland, Amsterdam (1985)

12. Diestel, J: Geometry of Banach Spaces - Selected Topics. Lecture Notes in Math., vol. 485. Springer, Berlin (1957)

13. Goebel, K, Reich, S: Uniform Convexity, Hyperbolic Geometry, and Nonexpansive Mappings. Series of Monographs and Textbooks in Pure and Applied Mathematics, vol. 83. Dekker, New York (1984)

14. Bridson, M, Haefliger, A: Metric Spaces of Non-positive Curvature. Springer, Berlin (1999)

15. Alfuraidan, MR, Khamsi, MA: Fixed points of monotone nonexpansive mappings on a hyperbolic metric space with a graph. Fixed Point Theory Appl. 2015, 44 (2015). doi:10.1186/s13663-015-0294-5

16. Bachar, M, Khamsi, MA: Fixed points of monotone mappings and application to integral equations. Fixed Point Theory Appl. 2015, 110 (2015). doi:10.1186/s13663-015-0362-x

17. Turinici, M: Fixed points for monotone iteratively local contractions. Demonstr. Math. 19, 171-180 (1986)

18. Turinici, M: Ran and Reurings theorems in ordered metric spaces. J. Indian Math. Soc. 78, 207-214 (2011)

19. Browder, FE: Nonexpansive nonlinear operators in a Banach space. Proc. Natl. Acad. Sci. USA 54, 1041-1044 (1965)

20. Göhde, D: Zum Prinzip der kontraktiven Abbildung. Math. Nachr. 30, 251-258 (1965)

21. Kirk, WA: A fixed point theorem for mappings which do not increase distances. Am. Math. Mon. 72, 1004-1006 (1965)

22. Menger, K: Untersuchungen über allgemeine Metrik. Math. Ann. 100, 75-163 (1928)

23. Reich, S, Shafrir, I: Nonexpansive iterations in hyperbolic spaces. Nonlinear Anal. 15, 537-558 (1990)

24. Busemann, H: Spaces with non-positive curvature. Acta Math. 80, 259-310 (1948)

25. Kirk, WA: Fixed point theory for nonexpansive mappings. In: Fixed Point Theory. Lecture Notes in Math., vol. 886, pp. 484-505. Springer, Berlin (1981)

26. Kirk, WA: A fixed point theorem in CAT(0) spaces and $\mathbb{R}$-trees. Fixed Point Theory Appl. 2004(4), 309-316 (2004)

27. Leustean, L: A quadratic rate of asymptotic regularity for CAT(0)-spaces. J. Math. Anal. Appl. 325, 386-399 (2007)

28. Khamsi, MA, Khan, AR: Inequalities in metric spaces with applications. Nonlinear Anal. TMA 74, 4036-4045 (2011)

29. Khamsi, MA: On metric spaces with uniform normal structure. Proc. Am. Math. Soc. 106, 723-726 (1989)

30. Ishikawa, S: Fixed points and iteration of a nonexpansive mapping in a Banach space. Proc. Am. Math. Soc. 59, 65-71 (1976)

31. Krasnoselskii, MA: Two observations about the method of successive approximations. Usp. Mat. Nauk 10, 123-127 (1955) 
32. Goebel, K, Kirk, WA: Iteration processes for nonexpansive mappings. Contemp. Math. 21, 115-123 (1983)

33. Garkavi, AL: The best possible net and the best possible cross-section of a set in a normed space. Transl. Am. Math. Soc. 39, 111-132 (1964). Translation from Izv. Akad. Nauk SSSR, Ser. Mat. 26, 87-106 (1962)

34. Zizler, V: On some rotundity and smoothness properties of Banach spaces. Diss. Math. 87, 415-440 (1971)

Submit your manuscript to a SpringerOpen ${ }^{\circ}$ journal and benefit from:

- Convenient online submission

- Rigorous peer review

- Immediate publication on acceptance

Open access: articles freely available online

- High visibility within the field

- Retaining the copyright to your article 